\title{
Early Detection of Autism in the Population
}

John D. McLennan M.D., Ph.D.

Follow this and additional works at: https://jdc.jefferson.edu/jeffjpsychiatry

Part of the Psychiatry Commons

Let us know how access to this document benefits you

\section{Recommended Citation}

McLennan, John D. M.D., Ph.D. (1998) "Early Detection of Autism in the Population," Jefferson Journal of Psychiatry. Vol. 14 : Iss. 1 , Article 2.

DOI: https://doi.org/10.29046/JJP.014.1.001

Available at: https://jdc.jefferson.edu/jeffjpsychiatry/vol14/iss1/2

This Article is brought to you for free and open access by the Jefferson Digital Commons. The Jefferson Digital Commons is a service of Thomas Jefferson University's Center for Teaching and Learning (CTL). The Commons is a showcase for Jefferson books and journals, peer-reviewed scholarly publications, unique historical collections from the University archives, and teaching tools. The Jefferson Digital Commons allows researchers and interested readers anywhere in the world to learn about and keep up to date with Jefferson scholarship. This article has been accepted for inclusion in Jefferson Journal of Psychiatry by an authorized administrator of the Jefferson Digital Commons. For more information, please contact: JeffersonDigitalCommons@jefferson.edu. 


\title{
Early Detection of Autism in The Population
}

\author{
John D. McLennan, M.D., M.P.H.
}

\begin{abstract}
Frequently there is a delay in the diagnosis of autism in children which may result in a lost opportunity to provide early intervention. Signal detection studies have suggested that single items have good predictability for autism which suggests simple screening procedures may be helpful in the early detection of an autistic disorder. Studies of autism in the very young have found that specific social deficits appear to characterize this group. A three stage procedure is presented as a possible approach to detect autism at an early age in the population thereby allowing for early intervention.
\end{abstract}

\section{INTRODUCTION}

Pervasive developmental disorders (PDD) in the Fourth Edition of the Diagnostic and Statistical Manual (DSM-IV) are characterized by severe and pervasive impairments in three domains: (i) reciprocal social interaction; (ii) communication and; (iii) behaviour, interests and activities (1). This paper focuses primarily on Autistic Disorder though some aspects may be applied to other disorders within the PDD spectrum. These disorders, particularly autism, constitute some of the most severe mental disorders of children.

Despite their severity and characteristic features, the diagnosis is often delayed, even until middle childhood, especially in high-functioning autistic individuals and when autism is co-morbid with severe mental retardation $(2,3)$. In one study, the diagnosis at first referral was made on average at 44 months, despite parents having had concerns on average at 17 months (4). Referrals to specialty clinics are rare before three years of age though there is now a trend for earlier referrals (2). There are several possible reasons for the delay in diagnosis including that: (i) primary healthcare workers are not specifically trained to detect autism early; (ii) current routine development screens do not alert primary care workers of possible autism as children are regularly screened for only motor, intellectual and perceptual development; (iii) the disorder is relatively rare; (iv) abnormalities in social and communicative development are difficult to assess in the pre-school period, (v) autism's presentation has large variation especially in the very young; and (vi) there is the lack of appropriate standardized screening instruments $(2,3)$.

There are several positive outcomes that could result from the early identification of autism. Early identification may (i) allow for early intervention while the nervous system is still malleable and responsive; (ii) enable education of the parents regarding developmental trajectories; (iii) determine the type of advice and services available; (iv) alleviate parental distress and provide support; (v) prevent inappropri- 
ate educational experiences for the child; and (vi) help prevent emotional and behavioural problems secondary to inappropriate treatment and expectations $(3,4,5)$.

Though there is no cure for pervasive developmental disorders, there are various treatments and resources for children and their families. Some studies have shown that early interventions, such as intensive behavioural programs, can improve the prognosis of this disorder $(6,7)$. Early initiation of programs which employ a high adult-to-child ratio and which contain a high degree of structure and continuity have also been found to be effective (8).

Clearly, early detection of autism in young children is essential. This paper reviews literature from several areas to develop recommendations for the early detection of autism in the population. The areas to be covered include (i) epidemiological studies of autism; (ii) signal detection procedures; (iii) features of autism in the very young; and (iv) previous attempts to screen for autism. This is followed by a possible procedure for early screening of autism in the population.

\section{EPIDEMIOLOGY OF AUTISM}

There is roughly a 20 fold range of prevalence figures for autism drawn from different studies. One of the lower figures is from an older U.S. study reporting $0.7 / 10,000$ (9). This contrasts with Ishii and Takahashi's Japanese study which reported $16 \cdot 0 / 10,000(10)$. A more typical rate is $4 / 10,000(11,12,13)$. The rates are significantly higher when other pervasive developmental disorders are included (e.g., 21/10,000) (14).

A Canadian epidemiological study of autistic syndromes involved a three stage design (15). The first stage screened all children 4 to 16 years of age within a circumscribed region $(\mathrm{N}=20,800)$. Screening questionnaires consisting of 19 items reflecting social, language, and repetitive behaviour and academic achievement were completed by teachers or counselors for children that were experiencing "problems or delays in any of the following areas: intellectual/academic, speech/language, behaviour/conduct or social/interpersonal." Stage two involved selecting cases based on total scores and patterns of the screening instrument and any who had "compelling examples of autistic like behaviour." In addition, various agencies were contacted to check for missing cases. Forty-six cases were identified and 35 of these were available for further assessment using: (i) new research diagnostic criteria; (ii) the Autism Behaviour Checklist; and (iii) psychometric data. Twenty-one of 35 met the criteria resulting in a prevalence of 10.1/10,000 (15).

Sugiyama and Abe followed up on referrals from a general screening performed on almost all 18 month old Japanese children in a specified district $(\mathrm{N}=11,320)(16)$. The 18 month assessment included a public health nurse using a developmental scale similar to the Denver and a pediatrician performing a physical, neurological, and developmental examination. Of the 168 referred for a second examination, 16 were diagnosed with autism by two child psychiatrists using operationalized criteria from the DSM-III following multiple evaluations at three month intervals. In most, the 
diagnosis was not finalized until 28.6 months of age. This resulted in a prevalence rate of $13 / 10,000(16)$.

\section{SIGNAL DETECTION}

Siegel and his group have used the signal detection statistical procedure to empirically identify a set of optimally efficient diagnostic criteria for the autistic disorder given a developmentally disabled differential diagnostic group $(17,18)$. This procedure takes into consideration sample size, prevalence of the disorder in the sample and prevalence of each criteria in the "diagnosis positive" group (17). This procedure simultaneously optimizes sensitivity and specificity and identifies the single criterion with the highest total predictive value. In other words, this procedure attempts to determine how well a single sign or symptom performs in identifying a syndrome in contrast to identifying all the elements of that syndrome. In the case of autism, they wanted to determine which of the 16 diagnostic criteria of DSM-III were most frequently evaluated as central to the diagnosis. The diagnostic criteria described as "marked lack of awareness of the existence or feelings of others," performed as well as the combination of criteria recommended by DSM-III-R. The second criteria with the next highest total predictive value was "persistent preoccupation with parts of objects" (18). The data collected for the DSM-III-R field trials for autistic disorder were re-analyzed using signal detection methods (18). Findings support inclusion of one diagnostic criterion ("marked lack of awareness of others") as mandatory, and four more (impaired imitation, abnormal social play, abnormal nonverbal communication, and abnormal speech) as alternate, associated criteria (18).

Oswald and Volkmar (19) used the signal detection procedure with the Autism Behavioural Checklist to detect the smallest number of items that contribute significantly to discriminating autism. The Autism Behaviour Checklist (ABC) is a 57 item instrument designed for teachers and is a component of the Autism Screening Instrument for Education Planning (20). The item that emerged as displaying the best combination of sensitivity and specificity in predicting the diagnostic group was the item, "Looks through people." This single item predicted the diagnostic group better than any of three different sum scores (19).

Mayes et al. (21) used the signal detection procedures to evaluate the power of individual items pulled from a chart review to discriminate Pervasive Developmental Disorder Not Otherwise Specified (PDDNOS) from autism and language disorders. Inability to make friendships was the most robust single predictor comparing PDDNOS to language disordered cases (21).

\section{FEATURES OF AUTISM IN THE VERY YOUNG}

Volkmar et al. (22) reviewed charts of 129 patients that had been diagnosed with infantile autism, PDD, atypical autism, or early childhood psychosis (DSM-III). Ninety-five percent of cases were recognized before age 30 months, $54.6 \%$ had an 
onset within the first year of life (22). In retrospective studies, some abnormalities have been reported in the first few months of life of autistic children, although it is unclear whether these are specific to autism, or to associated developmental delays (23).

Lord et al. (24) outlined some of the concerns of attempting to diagnose autism in very young children. One concern is that one of the core features of autism, communication abnormalities, is often assessed by detecting language abnormalities; however, very young children may not demonstrate sufficient language to assess for deviance, and nonverbal communication deviance can be difficult to measure in very young, nonverbal children. Social characteristics are another core component of the diagnosis of autism; however, differentiating deviance from delay can be difficult in very young children who do not show sufficient behaviour to allow qualitative judgment (e.g., peer relationships). In addition, behaviours, such as hand-flapping and other repetitive movements, that are seen as deviant in older autistic children may be relatively common in nonautistic children with mental ages below a year or 18 months (24).

To assess these concerns, Lord et al. (24) conducted a study using a semistructured interview instrument (Autism Diagnostic Interview-Revised) in the diagnosis of 51 autistic and 43-nonautistic mentally handicapped or language impaired preschool children of equivalent mental and chronological age. Sensitivity of an algorithm based on DSM-IV was high (96\%) and specificity was good (63\%) except for children with no expressive language and those with mental ages under 18 months. All but one autistic child met cutoff criteria in the three core domains of autism as did 16 of the nonautistic children. The main problem was the overdiagnosis of autism in young, severely mentally handicapped children (24).

DiLavore et al (25) and Baron-Cohen et al. (2), in reviewing their new instruments, discussed possible distinguishing features of early autism. Young autistic children have been found to have particular problems with:

(i) joint-attention tasks, especially the use of eye gaze, showing objects, and protodeclarative pointing (use of the index finger to indicate to another person an object of interest as an end in itself vs. protoimperative, that is, pointing to obtain an object);

(ii) delayed, absent or impoverished development of spontaneous, flexible imitation (particularly vocal or gestural imitation as opposed to imitation using objects or gross motor movements);

(iii) inability to use imitation as the basis of reciprocal interactions;

(iv) difficulties in spontaneous functional play and symbolic play (objects used as if they have other properties/identities) with young autistic children having particular difficulties in using a doll as an agent of action (in contrast to functional play and sensorimotor play that may be intact in the autistic child); and

(v) though attachments to parents/caregivers may develop, they may have disorganized behaviour in reaction to separation and reunion $(2,25)$. 
To assess these and other features of autism, DiLavore et al. (25) designed an observational instrument, the Pre-Linguistic Autism Diagnostic Observation Schedule, as a diagnostic tool for children less than 6 years of age and who are not yet using phrase speech. The protocol creates specific situations that allow for the observation of behaviours in each of the above interactional contexts (that is, joint attention, play, imitation, separation/reunion) (25). The instrument was found to have good reliability (kappas ranging from .62 to .89) and was clearly able to discriminate between autistic children (diagnosed by an experienced diagnostic team) and developmentally disabled children. Individual items that most discriminated autistic children from developmentally disabled children included the autistic children scoring more deviantly on repeating own actions when imitated, responding to joint attention, initiation of joint attention, social smile, and response to other's distress (25).

\section{SCREENING FOR AUTISM}

Johnson et al. (23) examined abnormalities in general screening tests which are routinely applied in the UK between 6 and 18 months of age by health visitors or general practitioners as predictors of later autism. These screens are brief assessments covering motor development, visual development, hearing and social development. They identified a group of autistic children and retrospectively assessed their infant screening records, as well as those of a group with learning difficulties (LD) and those of a random sample. A child was classified as having a problem in a category if the child was referred to a specialist, or if a note had been made to recheck the test at a later date, or if a note was made about the infant appearing unusual. At 6 months there were no statistically significant differences between groups and no groups had social problems which at that age would include features like lack of smiling or lack of social responsiveness to people. At 18 months the normal group had up to a $10 \%$ rate of abnormalities in vision and hearing but none in the social category. There were high rates in abnormalities in all categories for the LD group. The autistic group had a high frequency of abnormalities in the social category (23).

Baron-Cohen and his group (2) investigated the possibility of detecting autism at 18 months using a newly designed instrument, the Checklist for Autism in Toddlers (C.H.A.T.). They argued that both pretend play and joint attention behaviour are universal development achievements normally present in simple forms by 15 months and their absence on the routine 18 month screen could be clear specific indicators of autism or related disorders. The first part of the C.H.A.T. has questions for the parents and the second part has observations for the clinician. Examples from the first part include: "Does your child ever use his/her index finger to point to indicate interest in something" and "Does your child ever pretend, for example, to make a cup of tea using a toy cup and teapot, or pretend other things" (2).

They screened a high risk group, younger siblings of autistic children $(n=41)$, and compared them with a normal comparison group $(n=50)$. The C.H.A.T. was administered by a general practitioner or a health visitor. They found that key predictors of autism at 30 months were showing 2 or more of the following at 18 
months: lack of pretend play, lack of protodeclarative pointing, lack of social interest, lack of social play, or lack of joint-attention. The four children who failed two or more of the items at 18 months were diagnosed with autism at 30 months. In the normal comparison group, $80 \%$ passed all items and none failed more than one. The C.H.A.T. is now being studied in an epidemiological sample (2).

\section{DISCUSSION}

Both the Japanese and Canadian epidemiological studies screened entire circumscribed populations by utilizing stages to make the task feasible $(15,16)$. The Canadian study demonstrated a three stage process that was probably effective in obtaining most children with autism, but at the same time conserved the most comprehensive and expensive assessment for a relatively small group of children. However, the Canadian study used as its first stage the school system which would not be appropriate for an early detection intervention. The Japanese study was helpful in demonstrating the effective use of the primary care system at an early age. However, if this approach was adopted for a general protocol versus a circumscribed study, a three stage screening may be required as autism specialists would probably not be able to screen and subsequently treat the large numbers that might be referred in a two stage process. In their study, they comprehensively assessed 168 children from a group of 11,320 , that is $1.5 \%$ of the population.

Data from the signal detection studies highlight some variables that should probably be considered for a screening instrument. The four studies reviewed all found the most discriminating variable to be items assessing features of the social domain as opposed to language or stereotyped behaviour (17,18,19,21). A question asking about social relatedness ("marked lack of awareness of the existence or feelings of others" or "looks through' people") would seem most appropriate. However, the signal detection studies assessed a wide range of ages including adults when certain social deviant features may be easier to assess. In contrast, Lord et al.'s (26) large study of autistic persons with a wide range of chronological and mental ages found that multiple items were necessary to attain adequate sensitivity and specificity if samples with varying levels of language were considered. However, the sensitivity of the social reciprocity item was high even for the nonverbal group, making it a good screening question if false positives are not a particular concern (26). Applying the signal detection procedure to a particular young group would be helpful in identifying specific items for a screening instrument for this age group.

\section{RECOMMENDATIONS}

The following is an outline of a population screening procedure for early detection of autism taking into consideration the above review. Several assumptions need to be considered first. The first assumption is that the goal is to provide services for the most severely affected which are efficacious later. There is presently a multisite study attempting to replicate and expand on the encouraging positive 
outcome studies found in Lovaas' early intervention program (6,7). A second assumption is that further feasibility studies would be undertaken prior to the institution of widespread screening. Essential issues to address in a feasibility study include assessing sensitivity and specificity, appropriate cutoff scores and predictive validity of the screen. Although this would ideally be structured so that all children that could benefit from intervention services would receive them, realistically this needs to be balanced with finite service resource availability. Other essential issues include assessing the impact of false positives and negatives and determining the costs to the health system for introducing new screening. One concern with false positives is the potential fear and stress induced in caregivers with the suggestion that their child may have a severe and chronic mental disorder. False negatives become a concern if there is exclusive reliance on a screening procedure with no backup mechanism that would identify truly ill children missed at an early screening point. Cost assessment is essential given the increasing scrutiny of health care expenditures. Though it is difficult to estimate costs of a program that would be integrated into an existing system, potential cost savings of early intervention have been highlighted by Lovaas (6). Given these assumptions, the following approach is suggested to attempt early detection of autism in the general population.

A three stage procedure is recommended if a large population is to be screened. In stage one, a very brief and easy to administer instrument that could be combined with preexisting screening procedures would be essential to foster wide scale adoption at low cost. Screening could occur at the standard 18 month pediatric primary care checkup. A starting point would be the use of questions from the first part of the C.H.A.T., the parent questions, given that parents are usually the first to be concerned about development. If the parent could answer the screening questions directly into a computer in the waiting room, further time could be saved by having rapid results automatically generated during the same visit. The result would be entered into a predetermined algorithm that would suggest either continued periodic primary care assessments or the use of more lengthy and comprehensive instruments that would be assessed by the next stage. Those children scoring positively on the initial screen could be given instruments such as the Childhood Autism Rating Scale (C.A.R.S.) or the Autism Behavioural Checklist (A.B.C.). The initial screen and these more comprehensive instruments could be reviewed by a developmental nurse specialist who would determine the need to proceed to a comprehensive evaluation at a specialized center. Cutoff scores would guide the developmental nurse specialist in addition to consultation from a specialized center. At the specialized center, instruments such as the Autism Diagnostic Instrument and the Pre-Linguistic Autism Diagnostic Observational Schedules could be employed. At this third stage, a decision would be made as to whether the child would enter into a specialized early intervention program.

Given that the initial screens are based on parental reports, the costs would be kept low and unreasonable expectations would not made of busy primary caregivers. Since PDD is relatively rare, if cutoffs on screening instruments are not set too low, the coordinating nurse specialist could review multiple primary care sites, hence 
covering a large population. Mechanisms would need to be devised to attempt to track down those missing the screen and those whose parents are illiterate. Such mechanisms could be linked with special assistance and follow up programs used for those with incomplete immunizations. Since no reasonably specific screening instrument will be $100 \%$ sensitive, the system should be sufficiently flexible to have older children screened in alternative ways.

Overall, it appears that universal screening for the detection of early autism is not unreasonable. As autism constitutes one of the most severe mental disorders of childhood with high long term costs, it seems appropriate to seriously consider such a screening approach. If ongoing studies confirm the effectiveness of early intervention programs, neglect of early screening would be tragic.

\section{REFERENCES}

1. APA Task Force on DSM-IV: Diagnostic and Statistical Manual of Mental Disorders, Fourth Edition. Washington: American Psychiatric Association, 1994.

2. Baron-Cohen, S, Allen J, Gillberg C: Can Autism be detected at 18 months?: The needle, the haystack and the C.H.A.T. British Journal of Psychiatry 1992; 161:839-843.

3. Vostanis P, Smith B, Chung C, Corbett J: Early detection of childhood autism: a review of screening instruments and rating scales. Child: care, health and development 1994; 20:165-177.

4. Smith B, Chung M, Vostanis P: The path to care in autism: is it better now? Journal of Autism and Developmental Disorders 1994; 24:551-563.

5. First L, Palfrey J: The infant or young child with developmental delay. The New England Journal of Medicine 1994; 330:478-483.

6. Lovaas $\mathrm{O}$ : Behavioural treatment and normal educational and intellectual functioning in young autistic children. Journal of Consulting and Clinical Psychology 1987; 55:1-9.

7. McEachin J, Smith T, Lovaas O: Long-term outcome for children with autism who received early intensive behavioural treatment. American Journal of Mental Retardation 1993; 97:359-372.

8. Howlin P, Rutter M: Treatment of Autistic Children. Chichester: Wiley, 1987.

9. Treffert D: Epidemiology of Infantile Autism. Archives of General Psychiatry 1970; 22:431-438.

10. Tanoe Y, Oda S, Asano F, Kawashima K: Epidemiology of infantile autism in southern ibaraki, Japan: differences in prevalence in birth cohorts. Journal of Autism and Developmental Disorders 1988; 18:155-166.

11. Lotter V: Epidemiology of autistic conditions in young children. Social Psychiatry 1966; 1:124-137.

12. Ritvo E, Freeman B, Pingree C, et al.: The UCLA-University of Utah epidemiologic survey of autism: prevalence. American Journal of Psychiatry 1989; 146:194-199.

13. Fombonne E, du Mazaubrun C: Prevalence of infantile autism in four French regions. Social Psychiatry and Psychiatric Epidemiology 1992; 27:203-210.

14. Wing L, Gould J: Severe impairments of social interaction and associated abnormalities in children: epidemiology and classification. Journal of Autism and Developmental Disorders 1979; 9:11-29.

15. Bryson S, Clark B, Smith I: First report of a Canadian epidemiological study of autistic syndromes. J. Child Psychol. Psychiat. 1988; 29:433-445. 
16. Sugiyama T, Abe T: The prevalence of autism in Nagoya, Japan: a total population study. Journal of Autism and Developmental Disorders 1989; 19:87-96.

17. Siegel B, Vukicevic J, Elliott G, Kraemer H: The use of signal detection theory to assess DSM-III-R criteria for autistic disorder. J. Am. Acad. Child Adolesc. Psychiatry 1989; 28:542-548.

18. Siegel B, Vukicevic J, Spitzer R: Using signal detection methodology to revise DSM-III-R: re-analysis of the DSM-III-R national field trials for autistic disorder. J. Psychiat. Res. 1990; 24:293-311.

19. Oswald D, Volkmar F: Signal detection analysis of items from the Autism Behavior Checklist. Journal of Autism and Development Disorders 1991; 21:543-549.

20. Krug D, Arick J, Almond P: Behaviour checklist for identifying severely handicapped individuals with high levels of autistic behaviour. Journal of Child Psychology and Psychiatry 1980; 21:221-229.

21. Mayes L, Volkmar F, Hooks M, Cicchetti D: Differentiating Pervasive Developmental Disorder Not Otherwise Specified from Autism and Language Disorders. Journal of Autism and Developmental Disorders 1993; 23:79-90.

22. Volkmar F, Stier D, Cohen D: Age of recognition of pervasive developmental disorder. American Journal of Psychiatry 1985; 142: 1450-1452.

23. Johnson M, Siddons F, Frith U, Morton J: Can autism be predicted on the basis of infant screening tests? Developmental Medicine and Child Neurology 1992; 34:316-320.

24. Lord C, Storoschuk S, Rutter M, Pickles A: Using the ADI-R to diagnose autism in preschool children. Infant Mental Health Journal 1993; 14:234-252.

25. DiLavore P, Lord C, Rutter M: The pre-linguistic autism diagnostic observation schedule. Journal of Autism and Developmental Disorders 1995; 25:355-379.

26. Lord C, Pickles A, McLennan J, Rutter M, Bregman J, Folstein S, Fombonne E, Leboyer M, Minshew N: Diagnosing autism: analyses of data from the Autism Diagnostic Interview. In American Psychiatric Association (Eds.), DSM-IV Source Book. Washington, DC: American Psychiatric Association, (in press). 